\title{
A mechanism for glycoconjugate vaccine activation of the adaptive immune system and its implications for vaccine design
}

\author{
Fikri Y Avci ${ }^{1,2}$, Xiangming Li $^{3}$, Moriya Tsuji ${ }^{3} \&$ Dennis L Kasper ${ }^{1,2}$
}

\begin{abstract}
Glycoconjugate vaccines have provided enormous health benefits globally, but they have been less successful in some populations at high risk for developing disease. To identify new approaches to enhancing glycoconjugate effectiveness, we investigated molecular and cellular mechanisms governing the immune response to a prototypical glycoconjugate vaccine. We found that in antigen-presenting cells a carbohydrate epitope is generated upon endolysosomal processing of group B streptococcal type III polysaccharide coupled to a carrier protein. In conjunction with a carrier protein-derived peptide, this carbohydrate epitope binds major histocompatibility class II (MHCII) and stimulates carbohydrate-specific CD4 ${ }^{+} \mathrm{T}$ cell clones to produce interleukins 2 and 4 -cytokines essential for providing T cell help to antibody-producing B cells. An archetypical glycoconjugate vaccine that we constructed to maximize the presentation of carbohydrate-specific T cell epitopes is 50-100 times more potent and substantially more protective in a neonatal mouse model of group B Streptococcus infection than a vaccine constructed by methods currently used by the vaccine industry. Our discovery of how glycoconjugates are processed resulting in presentation of carbohydrate epitopes that stimulate CD4 ${ }^{+} \mathrm{T}_{\text {cells }}$ has key implications for glycoconjugate vaccine design that could result in greatly enhanced vaccine efficacy.
\end{abstract}

Pathogenic extracellular bacteria often express high-molecularweight capsular polysaccharides (CPSs) that coat the microbial surface. CPSs have been considered T cell-independent antigens ${ }^{1-5}$, primarily because, when used as vaccines, they induce specific IgM responses in wild-type and $\mathrm{T}$ cell-deficient mice without inducing much IgM-to-IgG switching ${ }^{3}$, fail to induce a booster response (that is, a secondary antibody response after recall immunization) and fail to induce sustained $\mathrm{T}$ cell memory ${ }^{4}$.

The advantages of glycoconjugate vaccines over pure glycans in inducing immune responses are well documented ${ }^{5}$. Covalent coupling of a T cell-independent CPS to a carrier protein yields a glycoconjugate that, when used to immunize mammals, elicits T cell help for B cells that produce IgG antibodies to the polysaccharide component ${ }^{5-11}$. Thus glycoconjugates induce polysaccharide-specific IgM-to-IgG switching, memory $\mathrm{B}$ cell development and long-lived T cell memory. Glycoconjugate vaccines have played an enormous part in preventing infectious diseases caused by virulent pathogens such as Haemophilus influenzae, Streptococcus pneumoniae and Neisseria meningitidis ${ }^{9,12}$. However, the immunogenicity of these glycoconjugates has been variable, and this variability has been attributed to the structure of the particular polysaccharide in a given construct ${ }^{13,14}$. In addition, in some populations at high risk for developing disease, such as the elderly or immunocompromised individuals, immunogenicity has been relatively poor ${ }^{5,9}$. The current hypothesis - that, in the context of MHCII, a peptide generated from glycoconjugates is presented to and recognized by $\mathrm{T}$ cells ${ }^{15}$
(Supplementary Fig. 1)-overlooks the strong covalent linkage of carbohydrates to proteins in glycoconjugate vaccines that is unlikely to be broken within the endosome $e^{3,5}$. This current hypothesis of peptide-only presentation has been promulgated mainly because proteins have generally been viewed as the only antigens presented by MHCII molecules to $\mathrm{T}$ cells. We considered whether $\mathrm{T}$ cells can recognize carbohydrates if they are covalently linked to another molecule (for example, a peptide) that allows MHCII to present the hydrophilic carbohydrate on the antigen-presenting cell (APC) surface. We hypothesized that $\mathrm{T}$ cell failure to respond to carbohydrates (for example, bacterial CPSs) is due to failure of these molecules to bind MHCII, not to T cell inability to recognize presented glycans. We tested this hypothesis to gain insight into the mechanisms involved in carbohydrate processing and presentation by MHCII and in subsequent T cell recognition of glycoconjugate vaccines. An understanding of the immune mechanisms involved in glycoconjugate immunization is crucial in the rational design of new-generation vaccines against emerging infections.

\section{RESULTS \\ MHCII-presented carbohydrate epitopes elicit T cell help}

We investigated the mechanisms underlying APC processing and presentation of glycoconjugates consisting of the type III polysaccharide of group B Streptococcus (GBSIII), a typical T cell-independent polysaccharide, coupled to a carrier protein or peptide such as ovalbumin (OVA), tetanus toxoid (TT) or ovalbumin peptide (OVAp).

${ }^{1}$ Channing Laboratory, Brigham and Women's Hospital, Harvard Medical School, Boston, Massachusetts, USA. ${ }^{2}$ Department of Microbiology and Immunobiology, Harvard Medical School, Boston, Massachusetts, USA. ${ }^{3} \mathrm{HIV}$ and Malaria Vaccine Program, Aaron Diamond AIDS Research Center, Affiliate of The Rockefeller University, New York, New York, USA. Correspondence should be addressed to D.L.K. (dennis_kasper@hms.harvard.edu). 
Figure 1 GBSIII-specific IgG secretion can be stimulated by $\mathrm{CD}^{+} \mathrm{T}$ cells recognizing carbohydrate epitopes. (a,b) Concentration of IgG antibody to GBSIII in BALB/c mice (four to six mice per group) primed (day 0) and boosted (day 14) with different antigen combinations, as measured by ELISA in serum obtained on day 21. Mice primed with III-OVA and boosted with unconjugated GBSIII and mice primed with unconjugated GBSIII and boosted with III-TT had significantly lower specific IgG levels than either mice primed and boosted with III-OVA or mice primed with III-OVA and boosted with III-TT ( $\left.{ }^{* * * P}<0.0001\right)$. None of the mice had detectable antibodies to either GBSIII or OVA before immunization (data not shown). Data represent means \pm s.e.m. a

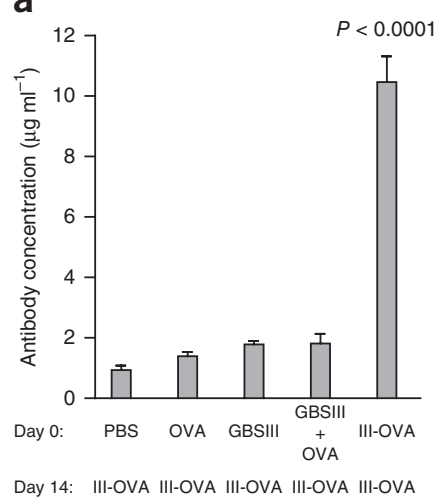

b

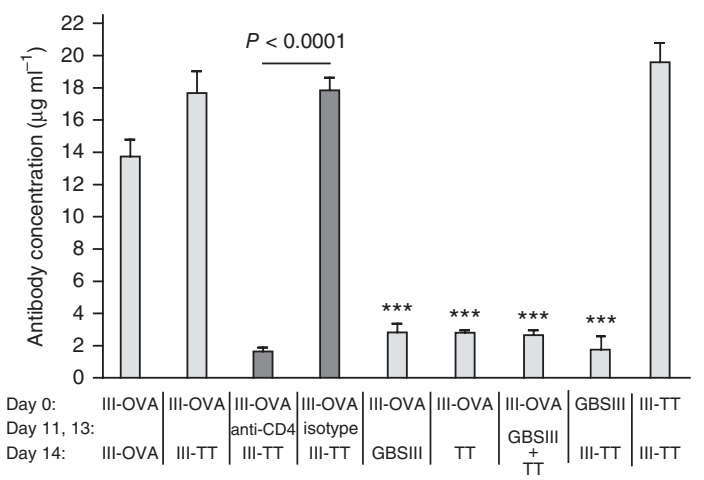

We first examined the adaptive immune response to glycoconjugates by priming mice with OVA and boosting them 2 weeks later with GBSIII conjugated to OVA (III-OVA). We compared polysaccharide-specific IgG levels in the sera of these mice with levels in the sera of mice both primed and boosted with the conjugate. Priming of naive mice with the carrier alone did not generate a robust secondary antibody response to the polysaccharide upon boosting with the glycoconjugate (Fig. 1a). However, mice primed and boosted with the glycoconjugate had strong IgG responses after recall vaccination (Fig. 1a). To determine whether the inability of OVA to induce a priming response for glycoconjugate boosting is due to a failure of $\mathrm{T}$ cell or $\mathrm{B}$ cell priming, we immunized mice with an unconjugated mixture of GBSIII and OVA, thereby providing B cells that had recent experience with GBSIII and T cells that had experience with presentation of the peptides derived from the OVA protein, and then boosted these mice with the glycoconjugate (Fig. 1a). After III-OVA recall immune stimulation, mice primed with GBSIII plus OVA-unlike III-OVA-primed mice-had essentially no secondary antibody response to the glycan (Fig. 1a). We measured OVA-specific IgG titers and GBSIII-specific IgG and IgM titers after only a priming dose of either GBSIII plus OVA or III-OVA. GBSIII-specific IgG was detectable only after priming of mice with III-OVA (Supplementary Fig. 2a). Serum levels of IgM antibody to GBSIII were similar in both groups of immunized mice, whether the glycan was conjugated or not (Supplementary Fig. 2b), an observation suggesting equivalent levels of carbohydrate-specific B cell priming. After priming, approximately the same level of OVA-specific IgG was measured in serum from both groups; this result suggested that OVA-specific T cell help was recruited after priming with either the GBSIII plus OVA mixture or the III-OVA glycoconjugate (data not shown). Additional control groups for this experiment involved mice primed with unconjugated GBSIII or with no antigen (PBS + alum) and boosted with III-OVA (Fig. 1a and Supplementary Fig. 2a,b).

In experiments examining whether $\mathrm{CD} 4^{+} \mathrm{T}$ cell recognition of a carbohydrate is a major factor in induction of the humoral immune response to glycoconjugates, we primed BALB/c mice with III-OVA, boosted with a conjugate comprising GBSIII and TT (III-TT), and measured serum levels of GBSIII-specific IgG (Fig. 1b). Control groups included mice primed and boosted with III-TT, primed and boosted with III-OVA, primed with GBSIII (unconjugated) and boosted with III-TT, primed with III-OVA and boosted with GBSIII (unconjugated), primed with III-OVA and boosted with GBSIII plus TT, and primed with III-OVA and boosted with TT. Boosting of IIIOVA-primed mice with III-TT induced GBSIII-specific IgG levels similar to those after priming and boosting with III-OVA (Fig. 1b).
These results strongly support recruitment of T cell help for induction of GBSIII carbohydrate-specific secondary immune responses via carbohydrate recognition.

Another possible explanation for our finding that type III polysaccharide-specific antibody response could be primed with III-OVA and boosted with III-TT is that activated B cells respond to III-TT without T cell help. We tested this possibility by boosting III-OVA-primed mice with III-TT after treatment with antibody to $\mathrm{CD} 4$ during the interval between priming and boosting. The excellent booster response observed in isotype control antibodytreated mice was abolished in CD4-specific antibody-treated mice (Fig. 1b). By flow cytometry, we found that there was complete depletion of $\mathrm{CD}^{+} \mathrm{T}$ cells from CD4-specific antibody-treated mice before secondary vaccination (Supplementary Fig. 2c). In addition, mice primed with III-OVA and boosted with GBSIII, TT or GBSIII plus TT had no booster response. These results led us to further examine the mechanisms by which $\mathrm{CD} 4^{+} \mathrm{T}$ cell recognition of GBSIII glycoconjugate vaccines could be mediated by the carbohydrate portion.

\section{Glycoconjugate carbohydrate is processed into smaller glycans}

To investigate the molecular and cellular mechanisms involved in immunization with GBSIII-containing glycoconjugates, we first examined glycoconjugate processing and presentation by APCs (for example, B cells or dendritic cells). Some CPSs are taken up by APC endosomes and depolymerized into smaller carbohydrates by oxidative agents such as reactive oxygen species (ROS) and reactive nitrogen species ${ }^{16,17}$. We assessed whether pure GBSIII (>100 kDa) is depolymerized within the APC endolysosome, as reported for Bacteroides fragilis polysaccharide $\mathrm{A}^{17}$. We incubated radiolabeled GBSIII (Supplementary Fig. 3) with Raji B cells for $18 \mathrm{~h}$ and isolated and lysed endolysosomes. We found by molecular sieve chromatography that the GBSIII within the endolysosome was significantly depolymerized, with a major peak at $\sim 10 \mathrm{kDa}$ (Fig. 2a). Western blot analysis showed that endolysosome preparations contained both the endosomal marker Rab5 and the lysosomal marker LAMP-1 (Supplementary Fig. 4). Comparison of the band density of CD19 (a cell surface protein)-labeled endolysosomal fractions with the band density of CD19-labeled, serially diluted cell surface fractions showed that endolysosomal fractions were essentially free $(\leq 5 \%)$ of cell surface content (data not shown).

As GBSIII and proteins are processed by different mechanisms in the endolysosome, we wondered whether the carbohydrate in the context of a glycoconjugate is also processed. We incubated Raji $\mathrm{B}$ cells with III-OVA, selectively radiolabeling only the polysaccharide 

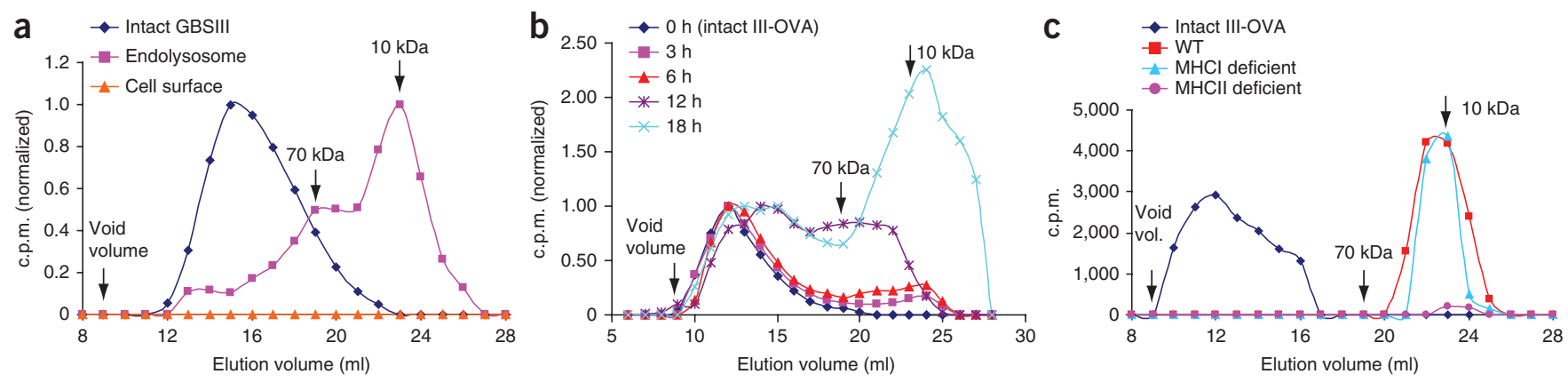

Figure 2 Processing and presentation of a glycoconjugate vaccine. (a) Superose 12 elution profile of lysates of Raji B cell endolysosomes and cell membranes after $18 \mathrm{~h}$ of incubation with unconjugated $\left[{ }^{3} \mathrm{H}\right] \mathrm{GBSIII}$. c.p.m., counts per minute. (b) Superose 12 elution profile of Raji B cell endolysosomes after incubation with $\left.{ }^{3} \mathrm{H}\right]$ III-OVA for $3,6,12$ or $18 \mathrm{~h}$. (c) Superose 12 elution profile of $\left[{ }^{3} \mathrm{H}\right] \mathrm{III}-\mathrm{OVA}$, obtained from surface extracts of mouse splenic mononuclear cells $(2 x$ $10^{8}$ ) after incubation with $\left[{ }^{3} \mathrm{H}\right] \mathrm{III}-\mathrm{OVA}$ and coimmunoprecipitation with antibody to $\mathrm{I}-\mathrm{A}^{\mathrm{b}}$.
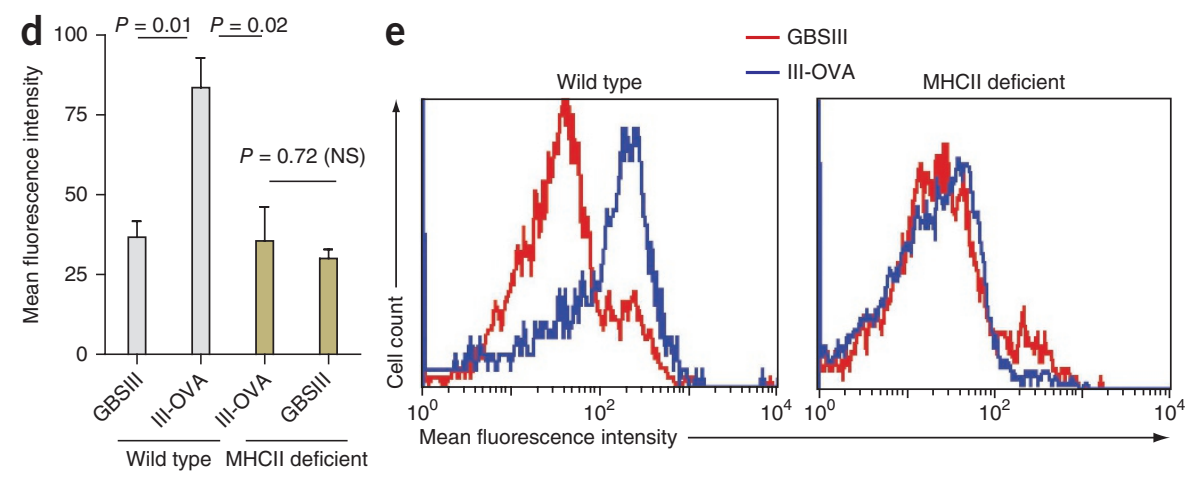

(d) Flow cytometric analysis of wild-type and MHCII-deficient bone marrow dendritic cells after incubation (18 h) with unconjugated GBSIII or III-OVA followed by surface staining of the cells with mAb to GBSIII. NS, not significant; WT, wild type. Data represent means \pm s.e.m. (e) Representative histogram for the experiment depicted in $\mathbf{d}$.

with ${ }^{3} \mathrm{H}$ (Supplementary Fig. 3). After $18 \mathrm{~h}$ of uptake and processing, $\left[{ }^{3} \mathrm{H}\right]$ III-OVA was degraded to a molecular size similar to that of pure unconjugated GBSIII after depolymerization (processed glycan

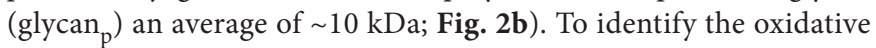
agents responsible for polysaccharide processing in III-OVA, we incubated Raji B cells with $\left[{ }^{3} \mathrm{H}\right] \mathrm{III}-\mathrm{OVA}$ in the presence of ROS inhibitors, detecting inhibition of polysaccharide processing by superoxide and hydroxyl radical inhibitors (Supplementary Fig. 5a,b, respectively) but not by a hydroperoxide inhibitor (Supplementary Fig. 5c).

\section{Processed carbohydrates are presented on the APC surface}

To determine whether MHCII-associated processed carbohydrates $\left(\right.$ glycan $_{\mathrm{p}}$ ) are presented on the APC surface, we conducted immunoprecipitation, flow cytometry and western blot experiments. First, we incubated Raji B cells with unconjugated pure GBSIII. Although $\left[{ }^{3} \mathrm{H}\right]$ GBSIII was endocytosed and processed to a smaller molecular size (Fig. 2a), immunoprecipitation of Raji B cell surface-membrane fractions with a monoclonal antibody $(\mathrm{mAb})$ to $\mathrm{MHCII}$ revealed no GBSIII on the cell surface in the context of MHCII (Fig. 2a). However, immunoprecipitation of APC surface membranes with mAb to MHCII after incubation of $\left[{ }^{3} \mathrm{H}\right]$ III-OVA with Raji B cells (Supplementary Fig. 6a) or mouse splenic mononuclear cells (Fig. 2c) showed surface co-precipitated, endosomally processed $\left[{ }^{3} \mathrm{H}\right] \mathrm{GBSIII}$ ( glycan $_{\mathrm{p}}$ ). Immunoprecipitation of Raji B cells with a monoclonal antibody to the type III polysaccharide after incubation with either $\left[{ }^{3} \mathrm{H}\right] \mathrm{III}-\mathrm{OVA}$ or $\left[{ }^{3} \mathrm{H}\right]$ III-TT showed the carbohydrate epitope on the cell surface in the context of human leukocyte antigen DR (HLA-DR) but not on MHCII-deficient Raji-derived RJ2.2.5 cells (Supplementary Fig. 6b). Western blot analyses of cell-surface and endolysosome fractions revealed that the Raji cell surface was essentially free of endosomal and lysosomal content (Supplementary Fig. 4). As controls for the experiments with antibody to HLA-DR, we immunoprecipitated cell surface membrane-solubilized extracts of Raji B cells with antibodies to LAMP-1 (a lysosomal protein) and CD19; we detected no radiolabeled carbohydrate in immunoprecipitates (Supplementary Fig. 6a). Immunoprecipitation with $m A b$ to HLA-DQ or HLA-DP did not result in detection of radiolabeled carbohydrate on Raji $B$ cell ${ }^{18}$ surfaces (data not shown). Splenocytes from C57BL/6 wild-type mice and various knockout strains were incubated with $\left[{ }^{3} \mathrm{H}\right] \mathrm{III}-\mathrm{OVA}$, and splenocyte cell surface extracts were immunoprecipitated with a $\mathrm{mAb}$ to mouse MHCII molecules IA and IE. Splenocytes from wildtype and MHCI-deficient $\left(B 2 m^{\text {tmlJae }}\right)$ mice had surface-associated GBSIII ( $10 \mathrm{kDa})$; cells from MHCII-deficient $\left(H 2-A b 1^{\text {tm1Gru}}\right)$ mice did not (Fig. 2c). These data indicate that a radiolabeled carbohydrate is detected in conjunction with MHCII on the APC surface only when it is conjugated to a carrier.

For flow cytometry, bone marrow-derived dendritic cells (BMDCs) from wild-type and MHCII-deficient mice were incubated with GBSIII or III-OVA for $18 \mathrm{~h}$ and then labeled at $4{ }^{\circ} \mathrm{C}$ with a GBSIIIspecific $\mathrm{mAb}$ (IgG2a) followed by fluorophore-conjugated secondary antibody to mouse IgG. Only membranes of III-OVA-incubated wildtype BMDCs were labeled with GBSIII-specific mAb (Fig. 2d,e).

Our results in Figure 2c-e show that endosomally processed carbohydrates are presented by the MHCII pathway only when covalently conjugated to carrier proteins. One possible explanation for this observation is that processed carbohydrate epitopes $\left(\right.$ glycan $\left._{p}\right)$ are presented by MHCII only when linked to an MHCII-binding peptide formed in the endolysosome by proteolytic digestion of the carrier protein

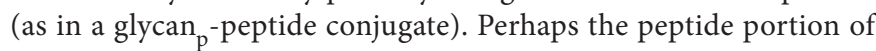

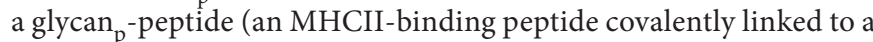
carbohydrate T cell epitope) binds MHCII, which carries the covalently linked carbohydrate to the APC surface. To assess whether glycan $_{\mathrm{p}}$-peptides created from the complex glycoconjugate vaccine are presented by MHCII, we conducted a western blot experiment 
with a glycoconjugate containing a single peptide epitope as the carrier (Supplementary Fig. 7a,b). In this vaccine, ovalbumin peptide epitope OVA323-339 (a T cell epitope of OVA ${ }^{19}$ ) was conjugated to GBSIII to form III-OVAp. The peptide was N-acetylated at its $\mathrm{N}$ terminus and extended with four amino acids at the $\mathrm{C}$ terminus to permit controlled conjugation to the polysaccharide. (OVAp can be coupled to only one aldehyde group on the sugar chain through its free amino group at the $\mathrm{C}$ terminus lysine residue.) In preliminary experiments (Supplementary Fig. 7b,c), this peptide readily bound MHCII on Raji B cells. Modifications of the peptide's amino acid composition did not affect its activation of the $\alpha \beta$ T cell receptor ( $\alpha \beta$ TCR); the modified peptide and the OVA323-339 peptide gave similar MHCII-restricted (for example, T cell activation blocked by $\mathrm{mAb}$ to $\left.\mathrm{I}-\mathrm{A}^{\mathrm{d}}\right) \mathrm{CD}^{+} \mathrm{T}$ cell proliferative responses in an in vitro mouse $\mathrm{T}$ cell proliferation assay (data not shown).

We incubated III-OVAp, pure GBSIII and pure OVAp with Raji B cells and examined cell surface-associated contents on western blots. Cell surface contents were collected, and solubilized by SDS sample

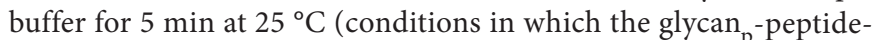
MHCII complex remained intact; see Supplementary Fig. 7). We blotted membranes with antibody to HLA-DR, antibody to GBSIII (capable of high-affinity reactions with polysaccharides as small as six repeating units) or antibody to OVAp. Immune complexes containing HLA-DR, GBSIII and peptide appeared in a band at $\sim 82 \mathrm{kDa}$ in membrane extracts from cells incubated with III-OVAp but not in those from cells incubated with unconjugated GBSIII (Supplementary Fig. 7a). HLA-DR $\alpha \beta$ dimers (self peptide-loaded or empty) were identified with $\mathrm{mAb}$ to HLA-DR at $\sim 64 \mathrm{kDa}^{20,21}$ (Supplementary Fig. 7a). The $\sim 18$-kDa difference in size (determined by protein markers) between unloaded HLA-DR and the glycan $_{\mathrm{p}}$-peptide-HLA-DR complex represents the approximate molecular size of the predicted glycan $_{\mathrm{p}}$-peptide, as carbohydrates migrate more slowly than proteins in gels $^{22}$. However, the mAb to GBSIII lightly bound to the overexpressed free MHCII at $\sim 64 \mathrm{kDa}$ (Supplementary Fig. 7a). To determine whether this interaction was nonspecific, we stimulated lysed naive Raji cells with OVAp and stained the transferred gel with mAb to GBSIII (Supplementary Fig. 7b). A light band at $64 \mathrm{kDa}$, but not at $\sim 82 \mathrm{kDa}$, was observed, suggesting that the $\sim 64-\mathrm{kDa}$ band in lane 5 of Supplementary Figure $7 \mathbf{a}$ was due to a nonspecific interaction. Human MHCII molecules (for example, HLA-DR10) have been shown to present OVAp ${ }^{23}$. It was crucial to show that OVAp could be presented by the MHCII molecules present on Raji B cells (whose HLA-DRB $1^{\star} 100101$ allele encodes HLA-DR10 (refs. 18,24)). We detected OVAp on a western blot with antibody to OVAp (Supplementary Fig. 7b), and we detected an OVAp-biotin conjugate when we examined by flow cytometry Raji cells (but not RJ2.2.5 cells) incubated with
OVAp-biotin and labeled with NeutrAvidin-fluorescein conjugate (Supplementary Fig. 7c). Our finding that OVAp binds Raji B cell MHCII molecules (Supplementary Fig. 7c) suggests that when III-OVAp

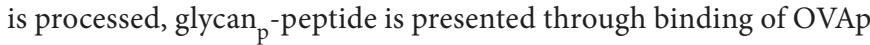
to MHCII (Supplementary Fig. 7a).

The immunoprecipitation and flow cytometry experiments (Fig. 2c-e) demonstrate that GBSIII glycan ${ }_{p}$ is presented on the cell surface in the context of MHCII only when conjugated to a carrier protein or peptide. Although the exact structural features of this complex must be defined by crystallography studies, western blot analysis (Supplementary Fig. 7a) suggests the possibility that a peptide portion of a glycan $_{\mathrm{p}}$-peptide binds $\mathrm{MHCII}$, enabling presentation of the glycan $p$ on the APC surface.

\section{Glycan $_{p}$ inhibits TCR OVAp recognition and antibody response}

We obtained lymphocytes from OVAp-specific TCR-transgenic mice (DO11.10) and wild-type mice (BALB/c) after immunization with III-OVAp or OVAp (two doses 2 weeks apart). In T cell proliferation experiments, we incubated irradiated splenic mononuclear cells (iAPCs) from wild-type naive BALB/c mice with $\mathrm{CD} 4^{+}$ $\mathrm{T}$ cells from either immune $\mathrm{BALB} / \mathrm{c}$ mice or immune DO11.10 mice (Fig. 3a-d). The iAPC and $\mathrm{CD} 4^{+} \mathrm{T}$ cell mixtures were stimulated in vitro with III-OVAp $\left(50 \mu \mathrm{g} \mathrm{ml}^{-1}\right)$, GBSIII $\left(37.5 \mu \mathrm{g} \mathrm{ml}^{-1}\right.$; equivalent GBSIII content to that in $50 \mu \mathrm{g} \mathrm{ml}^{-1}$ of III-OVAp), or OVAp $\left(12.5 \mu \mathrm{g} \mathrm{ml}^{-1}\right.$; equivalent OVAp content to that in $50 \mu \mathrm{g} \mathrm{ml}^{-1}$ of III-OVAp). As expected, unconjugated GBSIII did not stimulate proliferation of $\mathrm{CD}^{+}{ }^{+} \mathrm{T}$ cells from immunized $\mathrm{BALB} / \mathrm{c}$ or DO11.10 mice (Fig. 3a-d). However, CD $4^{+} \mathrm{T}$ cells from III-OVAp-immunized $\mathrm{BALB} / \mathrm{c}$ mice responded better to the glycoconjugate than to the peptide alone (Fig. 3a).

OVAp induced robust proliferation of DO11.10 CD4 ${ }^{+} \mathrm{T}$ cells from III-OVAp-immunized mice (stimulation index $=284.6$; Fig. $3 \mathbf{b}$ ) and OVAp-immunized mice (stimulation index $=277.6$; Fig. $3 \mathbf{d}$ ). As anticipated, $\mathrm{CD}^{+} \mathrm{T}$ cells from naive DO11.10 mice proliferated similarly strongly in response to OVAp (data not shown). DO11.10 CD4 ${ }^{+}$ T cells from III-OVAp-immunized mice (in the presence of iAPCs) were stimulated only minimally after coculture with III-OVAp (Fig. 3b). Proliferation was 97\% lower in DO11.10 T cells (from III-OVApimmunized mice) stimulated with III-OVAp (stimulation index =7.9; Fig. 3b) than in those stimulated with OVAp (stimulation index $=284.6$; Fig. 3b). These results support our findings (Fig. 2c-e and

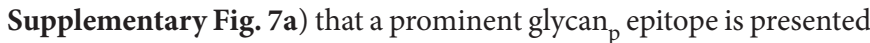
by iAPCs incubated with III-OVAp and that this carbohydrate is not recognized by the TCR of DO11.10 T cells (Fig. $\mathbf{3 b}$,d), which is, of course, specific for OVAp. Moreover, these findings suggest that the carbohydrate is masking presentation of OVAp on the APC surface.
Figure 3 T cells distinguish OVAp from III-OVAp, as presented by APCs. (a-d) Proliferation (as measured by $\left[{ }^{3} \mathrm{H}\right]$ thymidine incorporation) of $\mathrm{CD} 4^{+}$ $T$ cells obtained from wild-type BALB/C (a) or D011.10 (b) mice immunized with III-OVAp or wild-type BALB/c (c) or D011.10 (d) mice immunized with OVAp. T cells obtained from each group were stimulated in vitro with III-OVAp, OVAp or GBSIII in the presence of naive irradiated splenic mononuclear cells. Data are expressed as the mean stimulation index. Controls included

stimulation by GBSIII or no antigen; all negative controls had a stimulation index of $\sim 1$. (e) GBSIII-specific IgG concentrations on day 21 after

immunization with III-OVAp in D011.10 and WT BALB/c mice (same mice as in a,b). ND, not detectable. Data represent means \pm s.e.m. a

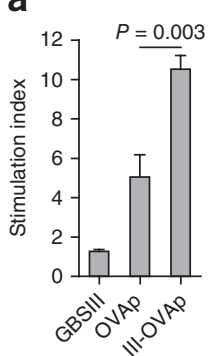

b

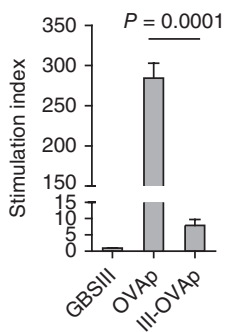

C

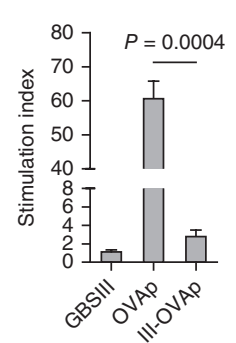

d

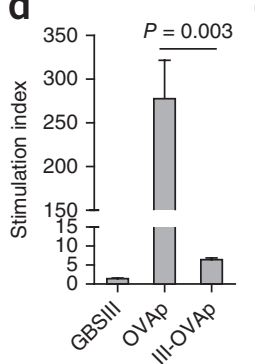

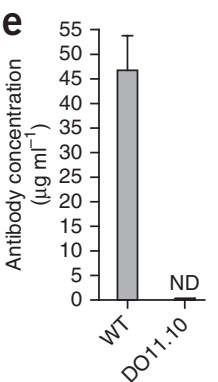


We hypothesize that the hydrophilic carbohydrate epitope is oriented between the MHCII-bound peptide and the TCR. Further characteri-

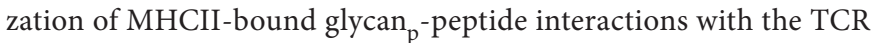
necessitates crystallography studies.

To determine whether the polysaccharide-specific IgG response is mediated by $\alpha \beta$ TCR glycan $p$ recognition upon glycoconjugate immunization, we measured GBSIII-specific IgG titers in DO11.10 and wild-type BALB/c mice immunized with III-OVAp. DO11.10 mice produced no, whereas wild-type mice developed high titers of GBSIIIspecific IgG (Fig. 3e). Neither WT nor DO11.10 mice had detectable antibodies to either GBSIII or OVAp before immunization (data not shown). DO11.10 and wild-type mice both developed GBSIIIspecific IgM, indicating that lack of IgG production in DO11. 10 mice was not due to lack of GBSIII-specific B cells (Supplementary Fig. 8). These results support the hypothesis that GBSIII recognition by helper T cells induces polysaccharide-specific IgG secretion by B cells. As DO11.10 mice lack the T cell repertoire recognizing the carbohydrate epitope of glycoconjugates, DO11.10 T cells could not induce polysaccharide-specific IgG secretion. In addition, DO11. 10 mice produced higher titers of OVAp-specific IgG than wild-type mice (Supplementary Fig. 8), an observation indicating that a global inability to produce IgG in DO11.10 mice does not account for their failure to produce GBSIII-specific IgG antibodies.

\section{$\mathrm{CD}^{+} \mathrm{T}$ cell clones recognize glycan $\mathrm{p}_{\mathrm{p}}$ in $\mathrm{MHCIl}$ context}

To investigate whether $\mathrm{CD} 4^{+} \mathrm{T}$ cells can recognize and interact with glycan $_{\mathrm{p}}$ presented by MHCII, we generated carbohydrate-specific mouse $\mathrm{CD}^{+} \mathrm{T}$ cell clones. We collected splenocytes from III-OVAimmunized BALB/c mice, enriched them for $\mathrm{CD} 4^{+} \mathrm{T}$ cells and cocultured them with iAPCs from spleens of naive BALB/c mice in the presence of III-TT for $6 \mathrm{~d}$. After purification of $\mathrm{CD} 4^{+} \mathrm{T}$ cells from these cocultured mixed cells, we cloned immune $\mathrm{CD} 4^{+} \mathrm{T}$ cells by limiting dilution. We re-stimulated the cloned cells at 12 -d intervals with III-OVA-pulsed iAPCs. We then conducted interleukin-2 (IL-2), IL-4 and interferon- $\gamma$ (IFN- $\gamma$ ) enzyme-linked immunospot (ELISPOT) assays to verify whether the clones recognized carbohydrate epitopes (Fig. 4). Two distinct CD4 ${ }^{+} \mathrm{T}$ cell clones secreted both IL-2 and IL-4 upon stimulation with GBSIII conjugated to any of three carrier proteins: OVA, TT or hen egg lysozyme (HEL). Neither clone responded to unconjugated carrier proteins alone, and neither produced IFN- $\gamma$ in the presence of GBSIII-containing glycoconjugates (data not shown). These data indicate the existence among $\mathrm{T}$ cells expanded with III-OVA and III-TT of cloned CD4 ${ }^{+} \mathrm{T}$ cells recognizing only the carbohydrate portion of the glycoconjugate.

To identify the restriction elements of these carbohydrate-specific clones, we added $\mathrm{mAbs}$ to MHCII proteins $\mathrm{I}-\mathrm{A}^{\mathrm{d}}$ and $\mathrm{I}-\mathrm{E}^{\mathrm{d}}$ to

Figure 4 Two $\mathrm{CD}^{+}{ }^{+} \mathrm{T}$ cell clones specifically recognize the carbohydrate portion of a glycoconjugate vaccine in the context of MHCII on APCs. (a-d) ELISPOT assays for the detection of $\mathrm{CD}^{+}{ }^{+} \mathrm{T}$ cell clones (clone $1(\mathbf{a}, \mathbf{b})$ and clone 2 (c,d) secreting IL-2 (a,c) and IL-4 (b,d)) conducted with several antigens, first in the absence and then in the presence of mAb to I-A $A^{d}$ or I-E (Anti-I-A $A^{d}$ or Anti-I-E $A b$ ). Irradiated naive mouse splenocytes were used as APCs. Data represent means \pm s.e.m. the ELISPOT assays. These antibodies completely inhibited $\mathrm{T}$ cell responses to both III-OVA and III-TT, as well as III-HEL (Fig. 4). Of note, $\mathrm{mAb}$ to I- $\mathrm{E}^{\mathrm{d}}$ inhibited IL- 2 and/or IL- 4 secretion by CD4 ${ }^{+}$ $\mathrm{T}$ cell clone 1 (Fig. 4a,b), whereas $\mathrm{mAb}$ to $\mathrm{I}-\mathrm{A}^{\mathrm{d}}$ inhibited the responses of $\mathrm{CD}^{+} \mathrm{T}$ cell clone 2 (Figs. $4 \mathbf{c}, \mathbf{d}$ ). Isotype controls for $\mathrm{mAb}$ to I- $\mathrm{A}^{\mathrm{d}}$ (IgG2b isotype) or mAb to I-E $\mathrm{E}^{\mathrm{d}}$ (IgG2a isotype) did not inhibit IL-2 or IL-4 production in either T cell clone (data not shown). These results indicate that $\mathrm{T}$ cell clones 1 and 2 recognize carbohydrate epitopes in the context of $\mathrm{I}-\mathrm{E}^{\mathrm{d}}$ and $\mathrm{I}-\mathrm{A}^{\mathrm{d}}$, respectively.

In an anti-TCR blocking experiment (Supplementary Fig. 9), we preincubated $\mathrm{CD} 4^{+} \mathrm{T}$ cell clones with various concentrations of Fab fragments of a mAb to either $\alpha \beta T C R$ or $\gamma \delta \mathrm{TCR}^{25}$. We incubated these cells with iAPCs in the presence of each antigen in culture medium and performed IL-2 ELISPOT assays $24 \mathrm{~h}$ later. The mAb to $\alpha \beta T C R$, but not that to $\gamma \delta \mathrm{TCR}$, inhibited activation of both T cell clones in a dose-dependent manner (Supplementary Fig. 9). Thus stimulation of $\mathrm{T}$ cell clones by carbohydrate epitopes is accomplished through the $\alpha \beta$ TCRs.

\section{An archetypical knowledge-based glycoconjugate construct}

We considered whether our insights into the mechanisms involved in activation of the adaptive immune system by glycoconjugate vaccines might guide the design and synthesis of a future generation of knowledge-based vaccines. The III-OVAp vaccine we designed for some of our mechanistic studies was useful in this regard. We calcu-

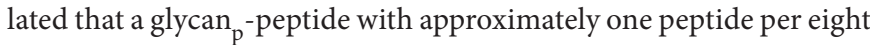
repeating units of polysaccharide could be created by depolymerization of GBSIII. This ratio would be likely to translate as a several-fold

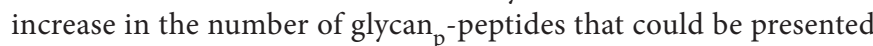
from each processed polysaccharide molecule over the number presented with standard glycoconjugates (Supplementary Fig. 10). The presentation of more carbohydrate epitopes per unit of vaccine could, in turn, enhance immunogenicity. We compared the immune response to this vaccine designed to maximize presentation of glycan epitopes $_{\mathrm{p}}$ (III-OVAp) with that to a glycoconjugate (III-OVA) constructed by the technology currently used in the industrial manufacture of several vaccines, including the GBS vaccines now in clinical trials (Fig. 5). Immunization of mice with III-OVAp (carbohydrate content, $0.2 \mu \mathrm{g}$ per dose) yielded GBSIII-specific IgG titers of $66 \mu \mathrm{g} \mathrm{ml}^{-1}$ - that is, $\sim 80$-fold higher than those following immunization with III-OVA at
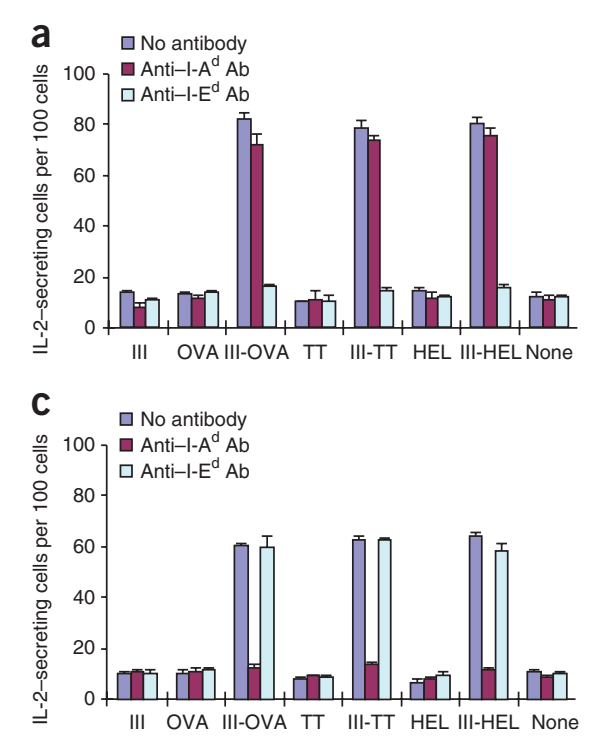
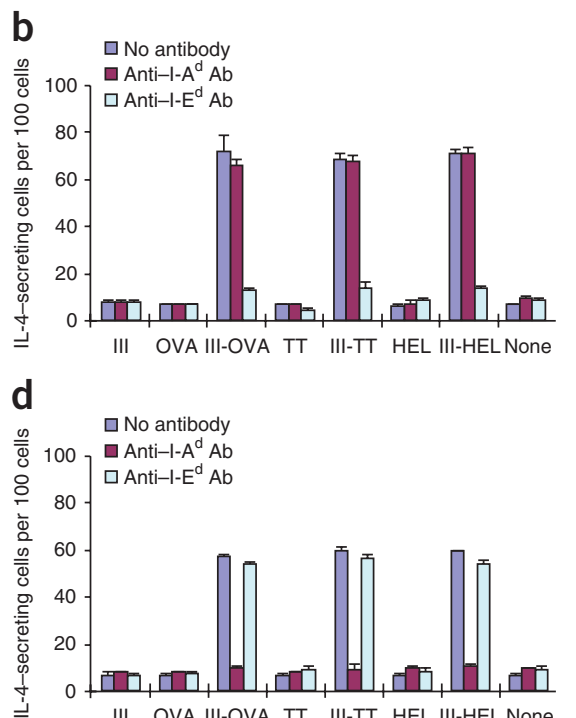
a

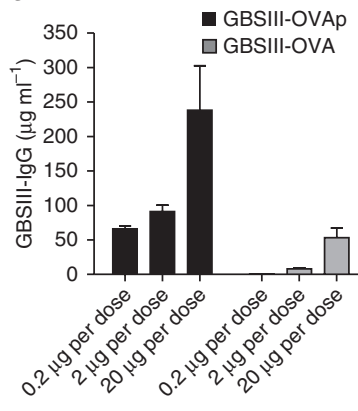

b

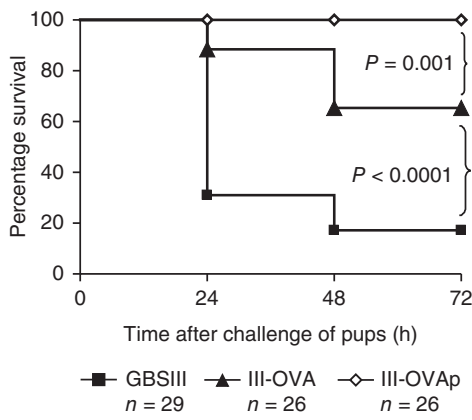

Figure 5 Immunization with III-OVAp induces a significantly stronger humoral immune response and greater protection than immunization with III-OVA. (a) GBSIII-specific IgG titers measured in serum obtained on day 35 after groups of BALB/c mice (six mice per group) were vaccinated three times (days 0,14 and 28 ) with three different doses $(0.2 \mu \mathrm{g}, 2 \mu \mathrm{g}$ or $20 \mu \mathrm{g}$; of carbohydrate) of either III-OVA or III-OVAp. (b) Survival of pups born to III-OVAp-, III-OVA- or unconjugated GBSIII-immunized dams and challenged with type III group B Streptococcus ( $n$ : number of challenged pups in each group). Data represent means \pm s.e.m.

the same carbohydrate dose (Fig. 5a). In fact, the IgG titer evoked by III-OVAp immunization with a carbohydrate dose of $0.2 \mu$ g per mouse was higher than that elicited in III-OVA-immunized mice by a 100fold greater carbohydrate dose $(20 \mu \mathrm{g}$ per mouse; Fig. 5a). Despite the marked difference in GBSIII-specific IgG titers ( 11-fold at the $2-\mu \mathrm{g}$ dose), GBSIII IgM titers were identical in III-OVAp-immunized and III-OVA-immunized mice (data not shown).

Higher IgG titers are not necessarily the only factor to consider in potential vaccine efficacy. Other factors include epitope specificity, affinity, avidity, subclass and functionality. Ultimately, however, the bottom-line predictor of vaccine efficacy is prevention of disease. These two vaccines were compared in a neonatal mouse protection assay (Fig. 5b) that is the current benchmark for GBS vaccine efficacy $^{26,27}$. Because severe GBS infection occurs primarily in the immediate postnatal period, vaccine-induced IgG antibodies in the mother must cross the placenta and protect infants postnatally. Simulating human vaccine delivery, we immunized adult female mice with III-OVA or III-OVAp ( $2 \mu \mathrm{g}$ of carbohydrate per dose); after vaccination, female mice were housed with males and impregnated. Pups ( $<36 \mathrm{~h}$ old) were challenged with five half-maximal lethal doses of live type III group B Streptococcus. All pups of III-OVAp-immunized mice survived, whereas only $65 \%$ of pups of III-OVA-immunized mice survived (Fig. 5b). Thus, the antibody levels achieved with this archetypical new-generation vaccine are correlated with efficacy in an animal model. This experiment suggests that knowledge of the mechanisms responsible for glycoconjugate processing and presentation permits the design of more efficacious vaccines.

\section{DISCUSSION}

The insights reported here raise the possibility of new glycoconjugate vaccines with chemical and physical properties specifically designed in light of information on antigen presentation. Until now, glycoconjugate construction has been a random process of linking two molecules (carbohydrate and protein) on the basis of an assumption that $\mathrm{T}$ cells don't recognize carbohydrates. The focus on achieving a chemical link between polysaccharide and protein by trial and error has necessitated lengthy, complex development efforts. Our new findings offer a rational explanation for how conjugates work and may render vaccine development a more straightforward and directed process.

Interactions between the mammalian immune system and protein antigens have been investigated for many years. More information has recently become available about key interactions of the adaptive immune system with nonprotein antigens. For instance, lipids and glycolipids are presented to T cells by MHCI-like CD1 family molecules (for example, CD1b and CD1d) ${ }^{28-31}$. Glycopeptides containing monosaccharides or small oligosaccharides generated by processing of natural glycoproteins and their synthetic derivatives (for example, type II collagen, HEL or viral nucleoprotein-derived glycopeptides) are recognized by $\mathrm{CD} 4^{+}$or $\mathrm{CD} 8^{+} \mathrm{T}$ cells ${ }^{32-37}$. Moreover, synthetic and natural glycopeptides containing tumor-associated mono- or oligosaccharides are recognized by $\mathrm{T}$ cells ${ }^{38,39}$. A number of synthetic vaccines comprising tumor-associated glycopeptides elicit humoral immune responses against cancer cells expressing tumor-associated carbohydrates $^{40}$. One class of complex carbohydrates (zwitterionic polysaccharides) activates $\mathrm{T}$ cells ${ }^{5,16}$ - an unexpected observation, given the immunologic paradigm categorizing carbohydrates as strictly $\mathrm{T}$ cell-independent antigens. The original hypothesis for glycoconjugate action ${ }^{3,15}$ was based on the failure of most pure polysaccharides to elicit IgG memory in mice. This paradigm presumes that elicitation of T cell help by glycoconjugates is attributable to MHCII presentation of peptides (derived by protein processing) to the TCR. In general, carbohydrates can be processed to smaller sizes in APC endolysosomes ${ }^{17}$ but fail to directly bind MHCII, are not presented to $\mathrm{T}$ cells, and consequently are indeed ' $\mathrm{T}$ cell independent ${ }^{2,5}$.

Several reports have suggested that attributing all of the T cell activation in glycoconjugate immunization to peptide presentation may reflect incomplete information. One group showed that infants vaccinated with $H$. influenzae type $\mathrm{b}(\mathrm{Hib})$ polysaccharide conjugated to the mutant diphtheria toxin protein $\mathrm{CRM}_{197}$ were primed for a secondary anti-Hib response to a Hib-TT conjugate ${ }^{41}$. This observation suggested the possibility of carbohydrate presentation to T cells, as the use of heterologous carriers did not interfere with the booster response. Another study explored how a purified pneumococcal glycoconjugate induces polysaccharide-specific memory, whereas intact pneumococci do not. These investigators suggested that covalent protein-polysaccharide bonding in a glycoconjugate-rather than a peptide alone-may be responsible for the $\mathrm{T}$ cell-dependent humoral immune response to the polysaccharide. This finding is consistent with our data showing that carbohydrate presentation to $\mathrm{CD} 4^{+} \mathrm{T}$ cells in the context of a covalently bound peptide can induce polysaccharide-specific adaptive immune responses. Supporting our findings, a recent report based on confocal microscopy images suggests the possibility of localization of the carbohydrate component of a pneumococcal glycoconjugate to the APC surface ${ }^{42}$.

The specific conditions used here (single carrier, conjugate, conjugation chemistry and one well-defined, MHC-restricted glycan $_{\mathrm{p}}$-peptide tested in inbred mice) will need to be expanded upon to generate relevant vaccines for clinical use. Other facets of the immune response to glycoconjugates that require study include the role of B cell subsets with different functions (for example, B1 and marginal-zone B cells) and the role of B cell maturation. A key issue is whether the same adaptive immune response to these polysaccharides can be expected in humans.

We summarize our findings in our new working model (Fig. 6). We discovered that there are $\mathrm{T}$ cell populations that recognize carbohydrate epitopes derived by APC processing of conjugate vaccines and that, when presented by MHCII, these epitopes recruit $\mathrm{T}$ cell help for the induction of adaptive immune responses to these vaccines. Understanding interactions between glycan $_{\mathrm{p}}$-peptide, MHCII and TCR at the structural level is crucial. 
Figure 6 Mechanism of T cell activation by glycoconjugate vaccines: a new working model. A schematic representation showing the steps in antigen processing and presentation of glycoconjugate vaccines resulting in helper $\mathrm{CD}^{+} \mathrm{T}$ cell induction of $\mathrm{B}$ cell production of IgG antibodies to the polysaccharide. The carbohydrate portion of the glycoconjugate binds to and cross-links the $\mathrm{B}$ cell receptor (BCR). The glycoconjugate is internalized into an endosome of the B cell. The carbohydrate portion of this glycoconjugate is processed in the endolysosome by ROS into saccharides composed of smaller numbers of repeating units than the full-length polysaccharide used in construction of the vaccine. The protein portion is processed by acidic proteases into peptides. Processing of both the protein and the carbohydrate portions of glycoconjugates

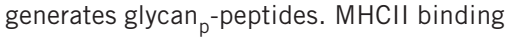
of the peptide portion of the glycan -peptide allows the presentation by $\mathrm{MHCll}$ of the more hydrophilic carbohydrate to the $\alpha \beta$ receptor of $C D 4^{+} T$ cells ( $\alpha \beta T C R$ ). The $\alpha \beta$ receptor of $C D 4^{+}$T helper cells recognizes and responds to glycan presented in the context of MHCII. Activation of the T cell by the carbohydrate-MHCII, along with co-stimulation, results in T cell production of cytokines such as IL-4 and IL-2, which in turn induces maturation of the cognate B cell to become a memory B cell, with consequent production of carbohydrate-specific IgG antibodies.

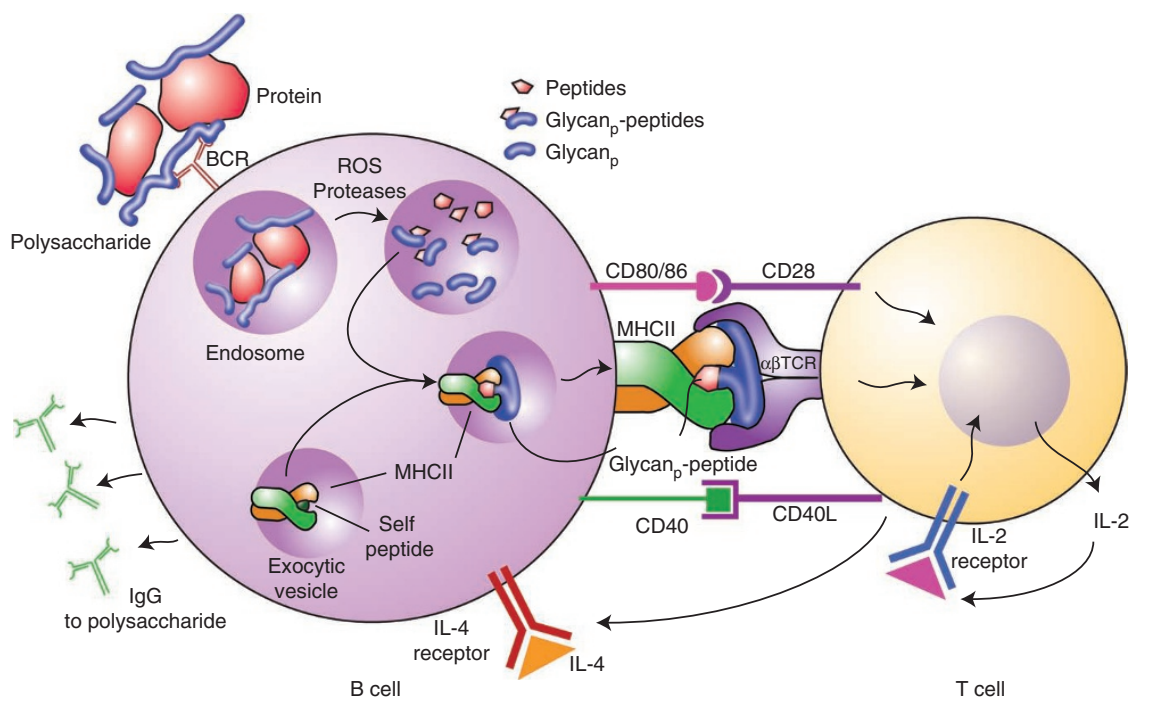

The data provided here map out a conceptual framework upon which to base future investigations of these interactions.

In short, this study shows that some of the variable immunogenicity previously considered polysaccharide specific may actually be related to the efficiency of carbohydrate presentation. New-generation vaccines with optimal, high-density presentation of carbohydrate epitopes could have a major role in prevention and control of many diseases. Glycans conjugated to proteins and lipids were recently cited as possibly "the most abundant structurally diverse class of molecules in nature." ${ }^{43}$. An understanding of the basic mechanisms governing glycoconjugate processing and presentation may be crucial to an understanding of immunity to microbial infections.

\section{METHODS}

Methods and any associated references are available in the online version of the paper at http://www.nature.com/naturemedicine/.

Note: Supplementary information is available on the Nature Medicine website.

\section{ACKNOWLEDGMENTS}

We thank B. Reinap and S. Mascuch for their valuable technical assistance and L. Paoletti for his advice. We also thank J. McCoy for her outstanding editorial assistance and T. DiCesare for his excellent artwork. This work was supported by funding from the following grants: US National Institutes of Health AI-089915, AI-070258 and AI-081510.

\section{AUTHOR CONTRIBUTIONS}

F.Y.A., M.T. and D.L.K. designed the research; F.Y.A. and X.L. performed the research; F.Y.A., X.L., M.T. and D.L.K. analyzed the data; and F.Y.A. and D.L.K. wrote the paper.

\section{COMPETING FINANCIAL INTERESTS}

The authors declare no competing financial interests.

Published online at http://www.nature.com/naturemedicine/.

Reprints and permissions information is available online at http://www.nature.com/ reprints/index.html.

1. Coutinho, A. \& Moller, G. B cell mitogenic properties of thymus-independent antigens. Nat. New Biol. 245, 12-14 (1973).

2. Barrett, D.J. Human immune responses to polysaccharide antigens: an analysis of bacterial polysaccharide vaccines in infants. Adv. Pediatr. 32, 139-158 (1985).
3. Guttormsen, H.-K. et al. Cognate stimulatory B cell-T cell interactions are critical for T cell help recruited by glycoconjugate vaccines. Infect. Immun. 67, 6375-6384 (1999).

4. Guttormsen, H.K., Wetzler, L.M., Finberg, R.W. \& Kasper, D.L. Immunologic memory induced by a glycoconjugate vaccine in a murine adoptive lymphocyte transfer model. Infect. Immun. 66, 2026-2032 (1998).

5. Avci, F.Y. \& Kasper, D.L. How bacterial carbohydrates influence the adaptive immune system. Annu. Rev. Immunol. 28, 107-130 (2010).

6. Beuvery, E.C., Van Rossum, F. \& Nagel, J. Comparison of the induction of immunoglobulin $\mathrm{M}$ and $\mathrm{G}$ antibodies in mice with purified pneumococcal type III and meningococcal group $\mathrm{C}$ polysaccharides and their protein conjugates. Infect. Immun. 37, 15-22 (1982).

7. Schneerson, R., Barrera, O., Sutton, A. \& Robbins, J.B. Preparation, characterization and immunogenicity of Haemophilus influenzae type b polysaccharide-protein conjugates. J. Exp. Med. 152, 361-376 (1980).

8. Wessels, M.R. et al. Stimulation of protective antibodies against type la and lb group B streptococci by a type la polysaccharide-tetanus toxoid conjugate vaccine. Infect. Immun. 61, 4760-4766 (1993).

9. Weintraub, A. Immunology of bacterial polysaccharide antigens. Carbohydr. Res. 338, 2539-2547 (2003).

10. Mitchison, N.A. T cell-B cell cooperation. Nat. Rev. Immunol. 4, 308-312 (2004).

11. Colino, J. et al. Parameters underlying distinct T Cell-dependent polysaccharidespecific IgG responses to an intact Gram-positive bacterium versus a soluble conjugate vaccine. J. Immunol. 183, 1551-1559 (2009).

12. Trotter, C.L. et al. Optimising the use of conjugate vaccines to prevent disease caused by Haemophilus influenzae type b, Neisseria meningitidis and Streptococcus pneumoniae. Vaccine 26, 4434-4445 (2008).

13. Leonard, E.G., Canaday, D.H., Harding, C.V. \& Schreiber, J.R. Antigen processing of the heptavalent pneumococcal conjugate vaccine carrier protein CRM197 differs depending on the serotype of the attached polysaccharide. Infect. Immun. 71, 4186-4189 (2003)

14. Kamboj, K.K., Kirchner, H.L., Kimmel, R., Greenspan, N.S. \& Schreiber, J.R. Significant variation in serotype-specific immunogenicity of the seven-valent Streptococcus pneumoniae capsular polysaccharide-CRM197 conjugate vaccine occurs despite vigorous T cell help induced by the carrier protein. J. Infect. Dis. 187, 1629-1638 (2003).

15. Janeway, C.A., Travers, P., Walport, M. \& Chlomchik, M. Immunobiology, 6th edn. Ch. 9 (Garland Science Publishing, New York, 2005).

16. Cobb, B.A., Wang, Q., Tzianabos, A.O. \& Kasper, D.L. Polysaccharide processing and presentation by the MHCII pathway. Cell 117, 677-687 (2004).

17. Duan, J., Avci, F.A. \& Kasper, D.L. Microbial carbohydrate depolymerization by antigen-presenting cells: deamination prior to presentation by the $\mathrm{MHCll}$ pathway. Proc. Natl. Acad. Sci. USA 105, 5183-5188 (2008).

18. Bentley, G. et al. High-resolution, high-throughput HLA genotyping by nextgeneration sequencing. Tissue Antigens 74, 393-403 (2009).

19. McFarland, B.J., Sant, A.J., Lybrand, T.P. \& Beeson, C. Ovalbumin (323-339) peptide binds to the major histocompatibility complex class II I-A protein using two functionally distinct registers. Biochemistry 38, 16663-16670 (1999). 
20. Hitzel, C., Grüneberg, U., van Ham, M., Trowsdale, J. \& Koch, N. Sodium dodecyl sulfate-resistant HLA-DR "superdimer" bands are in some cases class II heterodimers bound to antibody. J. Immunol. 162, 4671-4676 (1999).

21. Kalka-Moll, W.M. et al. Zwitterionic polysaccharides stimulate T cells by MHC class II-dependent interactions. J. Immunol. 169, 6149-6153 (2002).

22. Krinos, C.M. et al. Extensive surface diversity of a commensal microorganism by multiple DNA inversions. Nature 414, 555-558 (2001).

23. Shimojo, N. et al. Identification of the disease-related T cell epitope of ovalbumin and epitope-targeted T cell inactivation in egg allergy. Int. Arch. Allergy Immunol. 105, 155-161 (1994)

24. DeNardo, G.L. et al. Nanomolecular HLA-DR10 antibody mimics: a potent system for molecular targeted therapy and imaging. Cancer Biother. Radiopharm. 23, 783-796 (2008).

25. Tsuji, M. et al. $\gamma \delta \mathrm{T}$ cells contribute to immunity against the liver stages of malaria in $\alpha \beta$ T cell-deficient mice. Proc. Natl. Acad. Sci. USA 91, 345-349 (1994).

26. Paoletti, L.C. et al. Neonatal mouse protection against infection with multiple group B streptococcal (GBS) serotypes by maternal immunization with a tetravalent GBS polysaccharide-tetanus toxoid conjugate vaccine. Infect. Immun. 62, 3236-3243 (1994).

27. Paoletti, L.C. et al. Synthesis and preclinical evaluation of glycoconjugate vaccines against group B Streptococcus types VI and VIII. J. Infect. Dis. 180, 892-895 (1999).

28. Brigl, M. \& Brenner, M.B. CD1: antigen presentation and T cell function. Annu. Rev. Immunol. 22, 817-890 (2004).

29. Sieling, P.A. et al. Cd1-restricted T cell recognition of microbial lipoglycan antigens. Science 269, 227-230 (1995)

30. Moody, D.B. et al. Structural requirements for glycolipid antigen recognition by CD1b-restricted T cells. Science 278, 283-286 (1997).

31. Kawano, T. et al. CD1d-restricted and TCR-mediated activation of $\mathrm{V}_{\alpha} 14$ NKT cells by glycosylceramides. Science 278, 1626-1629 (1997)

32. Dzhambazov, B. et al. The major T cell epiope on type II collagen is glycosylated ion normal cartilage but modified by arthritis in both rats and humans. Eur. J. Immunol. 35, 357-366 (2005).
33. Deck, B., Elofsson, M., Kihlberg, J. \& Unanue, E.R. Specificity of glycopeptidespecific T cells. J. Immunol. 155, 1074-1078 (1995).

34. Mouritsen, S., Meldal, M., Christiansenbrams, I., Elsner, H. \& Werdelin, 0. Attachment of oligosaccharides to peptide antigen profoundly affects binding to major histocompatibility complex class-li molecules and peptide immunogenicity. Eur. J. Immunol. 24, 1066-1072 (1994).

35. Haurum, J.S. et al. Recognition of carbohydrate by major histocompatibility complex class I-restricted, glycopeptide-specific cytotoxic T-lymphocytes. J. Exp. Med. 180, 739-744 (1994).

36. Haurum, J.S. et al. Peptide anchor residue glycosylation: Effect on class I major histocompatibility complex binding and cytotoxic $T$ lymphocyte recognition. Eur. J. Immunol. 25, 3270-3276 (1995).

37. Abdel-Motal, U.M. et al. Immunization with glycosylated K-b-binding peptides generates carbohydrate-specific, unrestricted cytotoxic T cells. Eur. J. Immunol. 26, 544-551 (1996).

38. Gad, M., Werdelin, O., Meldal, M., Komba, S. \& Jensen, T. Characterization of $T$ cell hybridomas raised against a glycopeptide containing the tumor-associated T antigen, ( $\beta$ Gal (1-3) $\alpha$ GalNAc-O/Ser). Glycoconj. J. 19, 59-65 (2002).

39. Galli-Stampino, L. et al. T cell recognition of tumor-associated carbohydrates: The nature of the glycan moiety plays a decisive role in determining glycopeptide immunogenicity. Cancer Res. 57, 3214-3222 (1997)

40. Ingale, S., Awolfert, M., Gaekwad, J., Buskas, T. \& Boons, G.J. Robust immune responses elicited by a fully synthetic three-component vaccine. Nat. Chem. Biol. 3, 663-667 (2007)

41. Greenberg, D.P. et al. Enhanced antibody-responses in infants given different sequences of heterogeneous Haemophilus influenzae type-B conjugate vaccines. J. Pediatr. 126, 206-211 (1995).

42. Lai, Z. \& Schreiber, J.R. Antigen processing of glycoconjugate vaccines; the polysaccharide portion of the pneumococcal $\mathrm{CRM}_{197}$ conjugate vaccine co-localizes with $\mathrm{MHC}$ II on the antigen processing cell surface. Vaccine 27, 3137-3144 (2009).

43. Hart, G.W. \& Copeland, R.J. Glycomics hits the big time. Cell 143, 672-676 (2010) 


\section{ONLINE METHODS}

Mice and cell lines. We purchased wild-type (C57BL/6NTac), MHCI-deficient $\left(B 2 m^{t m 1 J a e}\right)$ and MHCII-deficient (H2-Ab1 $1^{\text {tmlGru}}$ ) mice (female, 6 to 8 weeks old) from Taconic Farms. We used Raji B cells and MHCII-deficient Raji cells (RJ2.2.5) for antigen presentation studies. We used wild-type BALB/c mice (either from Taconic Farms or from Jackson Laboratories) and OVAp-specific TCR transgenic mice (C-Cg- $\mathrm{Tg}(\mathrm{DO} 11.10) 10 \mathrm{Dl} / \mathrm{J})$ from Jackson Laboratories for immunization, and we used primary cells from the immunized mice in $\mathrm{T}$ cell assays. All mouse experiments were approved by Harvard Medical Area Standing Committee on Animals (animal protocol 866).

Antigens. We isolated and purified GBSIII from type III group B Streptococcus strain M781 (ref. 44). GBSIII was allowed to react with amine-containing peptides (for example, lysine) of OVA (Sigma), TT (North American Vaccine) or OVAp ( $N$-acetyl-ISQAVHAAHAEINEAGRESGK; Genscript) forming polysaccharide-protein or polysaccharide-peptide conjugates ${ }^{44}$.

Immunizations. We immunized groups of four to six mice intraperitoneally (i.p.) on days 0 and 14 with the antigen of interest mixed with $0.5 \mathrm{mg}$ of $\mathrm{Al}(\mathrm{OH})_{3}$ gel adjuvant. Mice receiving injections of $\mathrm{Al}(\mathrm{OH})_{3}$-only served as negative controls.

Measurement of specific serum antibodies. We bled mice from the tail vein (approximately drops of blood from each) on days 0,14 and 21 of the immunization protocols. We determined levels of GBSIII-specific or carrier-specific antibodies in dilutions of sera by solid-phase ELISAs as described previously ${ }^{3}$.

Immunoprecipitation. We incubated Raji B cells $\left(1 \times 10^{8}\right)$, RJ2.2.5 cells $\left(1 \times 10^{8}\right)$ or splenic mononuclear cells from wild-type, MHCI-deficient or MHCII-deficient mice $\left(2 \times 10^{8}\right)$ with $0.4 \mathrm{mg}$ of $\left[{ }^{3} \mathrm{H}\right] \mathrm{III}-\mathrm{OVA}$ for $15-18 \mathrm{~h}$. We isolated cell membrane fractions by differential centrifugation and solubilized at $4{ }^{\circ} \mathrm{C}$ with lysis buffer ${ }^{16}$. Supernatants obtained from Raji and RJ2.2.5 cells were incubated overnight with mixing at $4{ }^{\circ} \mathrm{C}$ in the presence of protein A agarose beads containing $25 \mu \mathrm{g}$ of mAb to HLA-DR (clone L243, BioLegend), HLA-DQ (clone HLADQ1, BioLegend), HLA-DP (clone HI43, BioLegend) or LAMP-1 (clone 25, BD Biosciences) or a polyclonal Ab to CD19 (Cell Signaling Technology). Cell membrane fractions obtained from mouse splenic mononuclear cells were incubated with protein $\mathrm{G}$ agarose beads containing $25 \mu \mathrm{g}$ of mouse I-A/I-E antibody (clone M5/114.15.12, BioLegend). After overnight incubation, we washed the beads and then boiled for $20 \mathrm{~min}$ in $10 \%$ SDS-3 M NaCl and analyzed the supernatants by Superose 12 chromatography.

GBSIII presentation by BMDCs. We incubated BMDCs from wild-type or MHCII-deficient mice for $18 \mathrm{~h}$ at $37^{\circ} \mathrm{C}$ with either GBSIII or III-OVA. After incubation, we washed cells five times with $1 \times \mathrm{PBS}$ and then labeled at $4{ }^{\circ} \mathrm{C}$ (to ensure cell surface labeling) with a GBSIII-specific mAb (IgG2a) followed by AlexaFluor647-conjugated secondary antibody to mouse IgG (Invitrogen). We tested surface staining by flow cytometry (FACSCalibur System).

In vitro $\mathrm{T}$ cell proliferation assays. We conducted mouse $\mathrm{T}$ cell proliferation assays as described previously ${ }^{17}$. In brief, irradiated splenic mononuclear cells
(iAPCs; $1 \times 10^{5}$ per well) from naive mice were cocultured for $4 \mathrm{~d}$ with $\mathrm{CD} 4^{+}$ $\mathrm{T}$ cells $\left(1 \times 10^{5}\right.$ per well $)$ purified from the spleens of each immunized mouse strain (Mouse CD4 Subset Column Kit, R\&D Systems) and were stimulated in vitro with the specified antigens (Fig. 3). Proliferation was measured by $\left[{ }^{3} \mathrm{H}\right]$ thymidine incorporation $8 \mathrm{~h}$ before harvesting.

T cell clone generation. We primed groups of $\mathrm{BALB} / \mathrm{c}$ mice subcutaneously (s.c.) with $4 \mu \mathrm{g}$ of III-OVA emulsified in complete Freund's adjuvant and boosted 3 weeks later by s.c. injection of $4 \mu \mathrm{g}$ of III-OVA emulsified with incomplete Freund's adjuvant. One week after boosting, we isolated lymphocytes from draining lymph nodes and cultured in vitro in the presence of III-TT $\left(100 \mu \mathrm{g} \mathrm{ml}^{-1}\right)$ for $6 \mathrm{~d}$. After this incubation, we purified CD4 ${ }^{+} \mathrm{T}$ cells with Lympholyte $\mathrm{M}$ (Cedarline Laboratories) and incubated for an additional $6 \mathrm{~d}$ in cDMEM, $10 \%$ FCS, and $10 \%$ T cell culture supplement. Meanwhile, splenocytes from III-OVA-immunized mice (two doses) were isolated and depleted of $\mathrm{CD}^{+} \mathrm{T}$ cells by beads coated with antibody to $\mathrm{CD} 3$ (Miltenyi) for use as APCs. These splenocytes $\left(3 \times 10^{6}\right)$ were irradiated and then cultured with $1 \times 10^{6}$ in vitro-expanded CD $4^{+} \mathrm{T}$ cells (see above) in the presence of III-TT $\left(100 \mu \mathrm{g} \mathrm{ml}^{-1}\right)$ for $6 \mathrm{~d}$. We then cloned these highly enriched $\mathrm{CD} 4^{+} \mathrm{T}$ cells by limiting dilution as described previously ${ }^{45}$.

ELISPOT assays. We co-cultured $\mathrm{CD} 4^{+} \mathrm{T}$ cell clones $\left(5 \times 10^{4}\right)$ with irradiated syngeneic splenocytes $\left(5 \times 10^{5}\right)$ in the presence of the specified antigens (Fig. 4). In some cases, we added a mAb $\left(50 \mu \mathrm{g} \mathrm{ml}^{-1}\right)$ to I- $\mathrm{A}^{\mathrm{d}}$ (BD Bioscience) or I-E (Biolegend) or an isotype control to the wells. Finally, we determined the relative numbers of antigen-specific $\mathrm{CD} 4^{+} \mathrm{T}$ cells secreting IL-2, IL- 4 or IFN- $\gamma$ by ELISPOT assays ${ }^{46}$. For the TCR blocking experiment (Supplementary Fig. 9), we preincubated cells from each $\mathrm{CD} 4^{+} \mathrm{T}$ cell clone for $30 \mathrm{~min}$ with the indicated concentrations of Fab fragments of mAb to either $\alpha \beta T C R$ (H57-597) or $\gamma \delta$ TCR (GL3), as previously performed ${ }^{25}$.

Neonatal protection assay. We used a maternal immunization-neonatal challenge model of GBS infection in mice to assess the protective efficacy of GBS vaccines $^{26,27}$. We vaccinated female BALB/c adult mice (Taconic) with GBSIII, III-OVA or III-OVAp (i.p. immunizations on days 0, 14 and 28). Mice were bred 1 week after receiving the third dose of vaccine. GBSIII cultures (strain M781) were injected i.p. (in a volume of $0.05 \mathrm{ml}$ ) into neonatal mouse pups ( $<36 \mathrm{~h}$ old). We assessed survival of pups 24,48 and $72 \mathrm{~h}$ after challenge.

Additional methods. Detailed methodology is described in the Supplementary Methods.

44. Wessels, M.R. et al. Immunogenicity in animals of a polysaccharide-protein conjugate vaccine against type III group B Streptococcus. J. Clin. Invest. 86, 1428-1433 (1990)

45. Tsuji, M., Romero, P., Nussenzweig, R.S. \& Zavala, F. CD4 ${ }^{+}$Cytolytic T cell clone confers protection against murine malaria. J. Exp. Med. 172, 1353-1357 (1990).

46. Tsuji, M. Assessing antigen-specific $\mathrm{CD} 8^{+}$and $\mathrm{CD} 4^{+} \mathrm{T}$ cell responses in mice afte immunization with recombinant viruses. Methods Mol. Med. 72, 361-367 (2002). 